\title{
La fécondité de la phénoménologie de la vie de Michel Henry pour les approches en deuxième personne
}

\author{
Un cas d'étude : le travail relationnel en urgence \\ psychiatrique
}

\section{Introduction}

"Je veux mourir, laissez-moi mourir!" Je viens de faire rentrer ce jeune homme de vingt-deux ans, et je reçois ces paroles comme un coup de massue. Je me dis immédiatement: "Qu'est-ce qu'il raconte, il ne peut pas mourir, je dois le rassurer: "Mais, non, il ne faut pas, regardez autour de vous, vos proches vous aiment, vous ne pouvez laisser votre enfant, et, en plus, vous avez un beau métier (il s'occupe d'enfants handicapés...).” "Et puis, voilà ce qui sort en fait de ma bouche: "Mais, bien sûr, vous avez le droit de mourir!" Qu'est-ce que je viens de lui dire? Pourquoi le conforter dans ce souhait inacceptable? Autant lui dire qu'il est libre de mourir!

La présente contribution prend son point de départ dans un étonnement: comment la force vitale peut-elle l'emporter sur le désir d'en finir? Quelles paroles, quel mode de relation à l'autre peuvent induire, soit l'un, soit l'autre? Comment l'intuition radicale déposée au cour de la phénoménologie de la vie offre-t-elle un levier théorique incroyable pour déjouer des mécanismes thérapeutiques parfois mortifères, et comment l'étude de situations d'urgence psychiatriques spécifiées peut-elle permettre de mettre en pratique de façon cruciale les enjeux philosophiques portés par la conception henryenne de la vie?

Tenter de répondre à ces interrogations fondamentales suppose quelques jalons théoriques (philosophiques, épistémologiques) et pratiques (thérapeutiques, d'urgence psychiatrique sur le travail de crise). On se demandera tout d'abord (I) comment la phénoménologie henryenne peut être appréhendée du point de vue d'une phénoménologie relationnelle qui place autrui au centre, alors même que l'auteur de L'essence de la manifestation a toujours refusé la transcendance de l'autre. Puis, on verra comment 
l'approche annoncée dans l'intitulé, qui mobilise les méthodologies en deuxième personne, nom de la relation à l'autre dans le cadre des sciences cognitives, peut être pertinente pour l'auteur de La barbarie, qui très tôt, dans le sillage de Husserl, dénonça l'objectivisme naturalisant des sciences. En nous confrontant à ces paradoxes, nous tâcherons de montrer comment le mécanisme de l'auto-affection est générateur d'une plastique relationnelle cohérente et nous le mettrons à l'épreuve d'une conception affinée de la deuxième personne en termes de "secondes personnes" (II). Depuis cette approche relationnelle de l'auto-affection, on comprendra mieux alors comment la phénoménologie henryenne de la vie offre un levier puissant pour une approche inédite du soin et de l'attention à la souffrance d'autrui (III). À partir de là, la description empirique des mécanismes relationnels vitalisants dans le travail de crise et d'urgence psychiatrique pourra tout à la fois vérifier certaines intuitions henryennes ontologiques et leur conférer une dynamique expérientielle individuée (IV).

\section{Michel Henry à l'écoute}

"En deuxième personne" est une expression couramment utilisée en sciences cognitives aujourd'hui pour décrire un mode de relation à autrui qui passe thématiquement par les catégories d'empathie et de sympathie, ${ }^{1}$ et qui nomme le statut de "CO-chercheur" de l'autre. Une telle approche permet de construire une objectivité requalifiée, non pas neutre et distanciée, bref abstraite, mais inscrite à même les subjectivités partagées, en d'autres termes, incarnée par la médiation de l'intersubjectivité. ${ }^{2}$

La philosophie de Michel Henry offre à première vue peu de prises à cet intérêt épistémologique contemporain pour les méthodes en deuxième personne: d'une part, il n'y a guère de place pour l'expérience d'autrui, semble-t-il, dans une phénoménologie de la vie où dominent les thèmes de

1 Cf. N. Depraz, "De l'empathie à la sympathie: de Husserl à Husserl (en passant par Lipps et Scheler), La phénoménologie historique 'en troisième personne' à l'épreuve de la phénoménologie expérientielle 'en première et en deuxième personne' ", (Colloque international, février 2009, Université de Rouen), dans J.-P. Cléro et P. F. Moreau (éds), La sympathie, à paraître.

2 Cf. à ce propos, N. Depraz, "Empathie et méthodologie en deuxième personne", Communication Powerpoint proposée dans le cadre du Colloque "Les paradoxes de l'empathie: entre émotion et cognition sociale", 15/16 janvier 2010, Université Paris-Ouest Nanterre La Défense, colloque organisé par A. Cukier et C. Malabou; N. Depraz et D. Cosmelli, "Empathy and openness: practices of intersubjectivity at the core of the science of consciousness", dans The problem of consciousness: new essays in phenomenological philosophy of mind, p. 163-203. Edited by Evan Thompson. Canadian Journal of Philosophy, supplementary volume 29, Calgary, Alberta: University of Calgary Press, 2003; N. Depraz, F. J. Varela et P. Vermersch, On becoming aware. A Pragmatics of Experiencing, Amsterdam-Boston, Benjamins Press, 2003, Première Section, chapitre 4, trad. fr. sous le titre À l'épreuve de l'expérience. Pour une pratique phénoménologique, Roumanie, Zeta Books, 2010. 
l'intériorité et de l'auto-affection; d'autre part, le phénoménologue radicalise par sa critique tous azimuts de l'objectivisme la suspicion initiale et récurrente des phénoménologues à l'égard de l'empirique et, plus largement, du discours et de la méthode scientifiques. On pourrait s'arrêter là : le débat semble tué dans l'œuf!

Mais, à y regarder de plus près, la perplexité se mue en curiosité: Michel Henry, sans mettre au premier plan l'altérité, a consacré au moins ${ }^{1}$ deux textes pour le moins saisissants à l'épreuve d'autrui en moi et pour moi, l'un, relativement tôt, "Pathos-avec", l'autre, très tard, "L'expérience d'autrui : phénoménologie et théologie ". Pourquoi ces articles sont-ils "saisissants"? D'une part, parce que l'auteur maintient une constance de pensée impressionnante qui se lit dans la reprise à l'identique de sa position vectrice d'un texte à l'autre: "toute communauté est invisible».3 Pour étayer cette position, l'auteur recourt à des situations expérientielles-type qui ont un caractère limite (la relation de la mère et de l'enfant, la communion des Saints), mais, loin d'y voir un problème pour la validité de son approche de l'intersubjectivité, leur caractère exceptionnel est de nature selon lui à offrir un levier radicalisant qui permet de nous affranchir d'une entente trop convenue, trop "naturelle", d'autrui. À tel point que le caractère foncièrement invisible de la relation à l'autre permet de rompre avec mon expérience la plus triviale d'un autrui qui serait simplement opposé à moi (séparé) ou, toutes choses égales, d'ailleurs, collé à moi (fusionné). On s’inscrit dès lors dans un régime inédit mais autrement plus passionnant de la réalité expérientielle "indivise", souche relationnelle commune de moi et de l'autre. Au fond, Michel Henry pense la relation à une telle profondeur expérientielle qu'il déroute nos habitudes les plus ordinaires. ${ }^{4}$ C'est à ce niveau de profondeur co-affective que l'on peut lire l'expérience érotique dont témoigne l'auteur dans Incarnation. Certes, ce qui y est décrit en termes d'échec de la relation érotique, ou encore du tragique fondamental de la dualité irréductible des flux pulsionnels ne saurait être purement et simplement gommé au profit d'un phénomène de l'eros appréhendé depuis sa dynamique immanente,

\footnotetext{
1 Je mentionne ici les deux articles connus et publiés par Michel Henry, sous réserve de l'existence d'autres écrits disponibles au Fonds Michel Henry de l'Université catholique de Louvain (Louvain-laNeuve) sous la responsabilité de J. Leclercq.

2 M. Henry, "Pathos-avec", Phénoménologie matérielle, Paris, PUF, "Epiméthée", 1990, p. 137-179; "L'expérience d'autrui: phénoménologie et théologie", Phénoménologie de la vie. Tome IV: Sur l'éthique et la religion, Paris, PUF, "Epiméthée", 2004, p. 155-163.

3 M. Henry, "Pathos-avec", art. cit., p. 166: "Devons-nous dire alors que, en dépit de l'apparence, toute communauté est invisible. Prenons ce risque"; "L'expérience d'autrui: phénoménologie et théologie", art. cit., p. 160: "toute communauté est par essence invisible. Elle a bien sûr (...) son 'apparaître' dans le monde, mais (...) cet apparaître n'est qu'une simple apparence coupée de la réalité."

$4 \mathrm{~A}$ ce propos, je me permets de renvoyer à N. Depraz, Le corps glorieux. Phénoménologie pratique de la Philocalie des Pères du désert et des Pères de l'Eglise, Bruxelles, Peeters, "Bibliothèque philosophique de Louvain", 2008, chapitre V.
} 
antérieure à la constitution des identités du moi et de l'autre. ${ }^{1}$ Mais je voudrais ici insister sur ce fait que les deux mouvements pulsionnels entrent en résonance, chacun se déployant et cédant à son tour, dans l'alternance de ses modalités actives et passives. ${ }^{2}$ M. Henry souligne ici la mobilité de la relation pulsionnelle, tout à la fois vibratoire et oscillatoire, qui n'est pas reconductible à une dualité figée ou à une mécanique biologique, s'alimentant bien plutôt à l'ouverture indécise de la relation elle-même.

Pour ce qui est du regard que l'auteur porte sur les sciences, il apparaît que, par delà la critique virulente de l'attitude standard du scientifique absorbé dans son objet et ce faisant aveugle à son ressenti et à son vécu de l'objet, ${ }^{3}$ Michel Henry ouvre un champ d'interrogations fascinantes pour les sciences du vivant, qu'il s'agisse de la biologie, de la médecine ou de la psychiatrie et, plus spécifiquement encore, de l'immunologie. L'intérêt explicite du phénoménologue pour la médecine et l'immunologie moléculaire laissent pressentir les potentialités de renouvellement de ses disciplines scientifiques déposées dans les concepts henryens d'auto-affection, de souffrance et d'ipséisation. ${ }^{4}$

Dans ces deux textes (sur lesquels je vais revenir en détail), ce qui est fascinant, c'est que l'auteur, tout en formulant avec constance ses réticences à l'égard de la possibilité pour la science de prendre en considération la connaissance sensible vivante, ressaisit avec une acuité extraordinaire les enjeux cruciaux de la dépression, du suicide, de l'auto-destruction, en faisant ressortir combien, au cœur de la crise ultime du sujet, celui-ci se "heurte à

1 J'ai développé ailleurs le caractère problématique de la conception henryenne de l'érotisme. Cf. N. Depraz, "Phénoménologie de la chair et théologie de l'eros", dans Michel Henry. Pensée de la vie et culture contemporaine. Colloque international de Montpellier, Paris, Beauchesne, 2006, p. 167-181. Je laisse ici de côté une telle critique, où dualité et résistance vont de pair; j'y reviendrai plus loin en lien avec l'expérience de la souffrance.

2 Incarnation. Une philosophie de la chair, Paris, Editions du Seuil, 2000, p. 301.

3 Evidemment radicale dans La barbarie (Paris, Grasset, 1987), encore très prégnante dans Incarnation et ré-affirmée avec force de la façon suivante: "Je reviens à la question du rapport science-éthique: et c'est pour dire qu'il n'y en a pas." Cette phrase lapidaire est citée par Florinda Martins dans son article "L'autre: le corps vivant" (dans Michel henry. Pensée de la vie et culture contemporaine, op. cit., p. 67, n. 1). Elle est extraite d'une Conférence inédite donnée par M. Henry à Nice en 1992, intitulée "Les sciences et l'éthique", et imprimée à l'occasion du Colloque de Montpellier en 2003. Je n'ai pas pu actuellement consulter cette Conférence, apparemment disponible sur le site http://alor.univ-montp3. fr/serpub.

4 Cf. les interventions de M. Henry, d'une part au Centre d'Etudes de philosophie de la Médecine (Institut Portugais d'Oncologie Francisco Gentil), intitulée "Douleur et souffrance. Subjectivité en clinique" (Cours d'Université d'été, Arrabida, 1997), prononcée à nouveau à la Faculté de Médecine de Paris en juin 2001, à l'occasion de la Vème Conférence internationale "Philosophie et psychiatrie", d'autre part à l'Institut de pathologie et immunologie moléculaire de l'Université de Porto le 14 septembre 2001, intitulée "Os outros em Eu", Editions IPATIMUP. "Les autres en moi: l'étrange, le terrible". Textes cités par F. Martins, art. cit., p. 68, n. 2, et publiés dans Phénoménologie de la vie, Paris, PUF, "Epiméthée", 2003, sous les titres respectifs "Souffrance et vie", p. 143-156, et "Eux en moi: une phénoménologie", p. 197-209. 
la cohérence interne de son être, à l'essence infrangible de la vie., ${ }^{1}$ Ainsi, de façon saisissante, en reprenant par exemple à son compte, dans le premier texte, l'auto-critique d'un scientifique, ${ }^{2}$ en en faisant ainsi un allié de sa quête radicale du vivant, il manifeste combien les scientifiques (plus que la science!), sont capables d'une lucidité remarquable. De même, dans le deuxième texte, sur un mode plus implicite mais dans une tonalité qui témoigne de sa sympathie profonde pour le travail des médecins, M. Henry formule avec une sobriété étonnante le vécu crucial du greffé. Il écrit que "l'autre doit être en nous", jusque dans cette injonction finale: "rendre une vie malade à son pouvoir et au bonheur de vivre. $\aleph^{3}$

Au fond, "Eux en nous" témoigne de l'intérêt profond du phénoménologue pour la relation à autrui et pour le travail des scientifiques, au fil conducteur d'une refonte fondamentale, et de la relation à l'autre, dont l'épreuve la plus cruciale se trouve être sise en moi-même, ${ }^{4}$ et de la relation à l'expérience la plus immédiate que connaissent les médecins et les malades au contact de la vulnérabilité radicale de leur identité en péril au moment de l'inscription de "l'autre" (du greffon, du foetus) en eux. ${ }^{5}$ L'écoute du phénoménologue est indéniable: les deux verrous qui semblent fermer l'accès à la possibilité d'interroger la fécondité de la phénoménologie de la vie pour les méthodes scientifiques en deuxième personne viennent de sauter. Nous pouvons à présent entrer en toute confiance sur ce terrain, à jamais "déminé».

1 M. Henry, "Souffrance et vie ", art. cit., p. 154.

2 Ibid., p. 143-144: “Selon François Jacob: "on interroge plus la vie aujourd'hui dans les laboratoires." (La logique du vivant, Paris, Gallimard, 1970, p. 320). En effet, on y étudie des processus matériels aveugles, homogènes à ceux qui forment le thème de la physique."

3 M. Henry, "Eux en nous ", art. cit., p. 205 et 209.

4 Dans Transcendance et incarnation. L'intersubjectivité comme altérité à soi chez Husserl (Paris, Vrin, 1995), j'ai montré comment la compréhension la plus radicale de l'expérience d'autrui invite à y ressaisir l'"altérité à soi " qui structure le sujet, ce qui résonne fortement avec la conception henryenne tardive d'autrui.

5 A propos de l'expérience de la greffe, cf. F. J. Varela, "Intimate distances: Fragments for a Phenomenology of Organ Transplantation", dans Journal of Consciousness Studies, 8, n5-7, 2001, p. 259-271 et, inspirée par cette expérience, N. Depraz, "The intimate other: A phenomenology of lucid embodiment in light of the lived experience of pregnancy", dans N. Depraz \& S. Gallagher (esd) Embodiment and awareness, Special issue de Theoria et Historia Scientiarum, Torun, 2003, p. 163-181, en français, dans une version revue, sous le titre: "L'autre intime. Phénoménologie de la grossesse", contribution à paraître dans le volume Les épreuves de la vie sous la direction de S. Camilleri et $\mathrm{Ch}$. Perrin. 


\section{La phénoménologie relationnelle de la deuxième personne aux «secondes 》 personnes : l'auto-affection comme relation}

On a vu que l'approche en deuxième personne dans le champ des sciences cognitives aujourd'hui permet de faire droit à autrui, dite "deuxième personne", en tant que sujet témoignant de son expérience "en première personne". La deuxième personne recèle cependant quelque ambiguité, dans la mesure où elle peut être appréhendée, si l'on suit D. Dennett sur ce point, comme un observateur distancié qui regarde de façon participative mais avec recul. J'ai ailleurs fait ressortir, en collaboration avec F. J. Varela et $\mathrm{P}$. Vermersch, une deuxième personne davantage située en "résonance" immédiate avec la première personne, dont les figures concrètes emblématiques sont le coach, la sage-femme, le thérapeute, plutôt que, comme D. Dennett y insiste, l'anthropologue-ethnologue, lequel correspond à une deuxième personne située en continuité avec la troisième. ${ }^{1}$

Aussi la deuxième personne apparaît-elle moins comme une instance ou un pôle distinct voire opposé aux autres pôles (1/3) qu'une dynamique qui se relie à l'un ou à l'autre. C'est ce constat qui nous a conduits à renommer cette dynamique depuis l'expression de "secondes personnes", de façon à mieux faire entendre, sous le pluriel, la dynamique graduelle et variative qui caractérise ce mode personnel essentiellement relationnel; de plus, le choix de l'adjectif qualificatif "second" plutôt que "deuxième", permet d'insister sur la facette, dans la deuxième personne, qui résonne directement avec la première, plutôt que sur l'objectivation qui porte la continuité avec la troisième. Pourquoi? Parce que, le "second" n'est jamais suivi d'un "troisième", le second interrompt la série des nombres, ce qui invite à se centrer sur la relation du "un" au "deux", tandis que "deuxième" ouvre une série et n'est qu'un maillon relatif, noyé dans la suite des nombres. ${ }^{2}$ A travers ce changement d'accent du "deuxième" au "second", on privilégie dans l'expérience d'autrui la continuité relationnelle profonde entre moi et l'autre, explicitement nommée par le terme de "résonance", qui souligne le continuum affectif de la relation. ${ }^{3}$

1 A propos de cette discussion, cf. N. Depraz, F. J. Varela et P. Vermersch, On becoming aware/A l'épreuve de l'expérience, op. cit., Section I, chapitre 2, 2.2. "Les structures de médiation: première, deuxième et troisième personne", 1. Contours des trois positions et 2. Multistratification des trois positions.

2 A ce propos, cf. N. Depraz et F. Mauriac, "Secondes personnes. Une anthropologie de la relation", Evolution psychiatrique, 2006.

3 Cf. N. Depraz et F. Mauriac, "La résonance comme épochè éthique", dans Alter. Revue de phénoménologie, $\mathrm{n}^{\circ} 13$, octobre 2005; F. Mauriac et N. Depraz, "Relationalität als methodischer Zugang zur Subjektivität", dans Psycho-logik 3, Methode und Subjektivität, K. Alber Verlag, p. 247-264, trad.

Varia 
On dispose, depuis cette expérience d'autrui située en résonance profonde avec moi que libèrent les "secondes personnes", un point d'appui tout à la fois méthodologique et ontologique pour éclairer d'un nouveau jour la conception henryenne de l'auto-affection. Ce faisant, on la lave de certaines apories dans lesquelles on la cantonne souvent, et on fait droit à plein à la conception radicale d'autrui que Michel Henry met en évidence depuis cette écoute à nouveaux frais de l'auto-affection. De quoi s'agit-il? De rien de moins que d'expérimenter l'auto-affection dans son sens absolu, c'est-à-dire le plus prégnant, comme la dimension la plus authentique de la relation. Ceci peut paraître osé voire contradictoire si l'on se fie au sens le plus obvie de l'auto-affection, qui depuis Kant (voire dans le sens tout d'abord relatif que lui confère Michel Henry dans L'essence de la manifestation) ne peut signifier qu'une expérience interne d'un sujet clos sur lui-même. C'est aussi le constat immédiat que l'on peut faire tout d'abord à la lecture d'un cas tout à la fois singulier et radical d'expérience intersubjective, celui de l'expérience érotique, dont on a déjà évoqué, depuis Incarnation, le caractère unilatéralement auto-érotique, à savoir solipsiste. L'eros henryen resterait pensé dans le cadre du paradigme de la conscience auto-affectée.

Cependant, cette lecture de l'auto-affection demeure superficielle. A cet égard, J.-L. Marion laisse entendre que l'auto-affection érotique de M. Henry fait sourdre un partage amical qui puise à une source affective commune: "[...] il subsiste un nombre impressionnant de textes de toutes époques, épars dans des revues, collectifs et actes de colloques, sous forme de libres entretiens ou de conférences très formelles. [...] On y découvre, derrière l'apparence auto-référentielle d'une globalité que rien ne semble entamer, qui marque indiscutablement les ouvrages majeurs, qu'en fait l'invention puissante de Michel Henry développait sa spontanéité créatrice à travers maints débats serrés et une infinité de rencontres privilégiées, sur un mode étonnamment dialogique. Cette entente, ce commerce et cette écoute surprenaient d'ailleurs souvent ceux qui en étaient restés à une acception d'abord superficielle de l'auto-affection. En fait, l'auto-affection n'avait rien d'un autisme, mais s'exerçait toujours par et dans une profonde affection, au double sens, indissolublement, du terme. Laffection par soi se laissait affecter elle-même par toutes les autres consciences auto-affectées que l'amitié lui attirait." Au fond, si l'eros henryen n'est en dernière instance ni autoréférentiel ni autiste mais éminemment dialogique comme l'affirme de façon intéressante J.-L. Marion, ce serait, non pas seulement dans l'expérience

fr.: "Phénoménologie de la relation", dans Psychiatrie, sciences humaines et neurosciences (revue), G. Granger éd., 2009.

1 Préface de J.-L. Marion à M. Henry, dans Phénoménologie de la vie, tome 1, De la phénoménologie, op. cit., p. 7-8. 
d'une "nappe affective souterraine " ${ }^{1}$ unique et fusionnelle à laquelle chacun viendrait boire, comme il l'écrit, plutôt, davantage, me semble-t-il, dans la résonance et le jeu alterné des pulsions en relation. C'est là, au cœur de la mobilité du jeu co-pulsionnel, qu'il convient de décrypter une telle affection mutuelle de soi par l'autre et de l'autre par soi. C'est dans cette conférence prononcée devant des médecins et des psychiatres que le phénoménologue avance le plus loin en direction d'une entente de la vie s'éprouvant elle-même en résonance avec une autre vie s'éprouvant elle-même, par où médecin et malade communiquent à un niveau affectif de profondeur telle que la distinction moi/autrui fait retraite au profit d'une unique continuité auto-affective relationnelle: alors que la douleur, locale, se concentre sur un aspect physique du corps susceptible d'être isolé, à savoir objectivé et, ce faisant, mis à distance, la souffrance se révèle globalement par elle-même et par elle seule, tient tout entière dans cette adhésion à elle-même, cet impossible écart qui fait du sujet un être-souffrant. Certes, il convient ici d'éviter tout nominalisme factice, dans la mesure où le "douloureux comme tel" ${ }^{2}$ retient tout autant cette immanence de l'être-souffrant: au-delà des mots, l'essentiel tient à l'expérience d'une coïncidence du sujet et du souffrir, qui définit l'auto-affection, et ouvre sur une entente à nouveaux frais de l'expérience d'autrui: si celle-ci "S'instaure sur le plan de la chair", et non des corps objectivés, c'est parce qu'elle se révèle depuis le moi entendu comme "rapport à soi", au sens d'épreuve de soi, première condition inhérente à cette expérience de l'autre comme "autre en nous". ${ }^{3}$

$\mathrm{Au}$ fond, ce qui est en jeu à travers cette conception radicale du soi comme altérité affective originaire, c'est le sens profond du vivant tel qu'il est généré par la vitalité de la relation à soi-même et se génère depuis lui-même dans sa propre croissance intérieure. On comprend mieux dès lors la profonde affinité ontologique entre les pensées de M. Henry et de F. Varela, leur entente commune de l'immanence du soi vivant, de son autonomie relationnelle (immanence structurée de l'auto-affection ou clôture opérationnelle de l'auto-poièse), leur scepticisme à l'égard de l'extériorité, leur conception dynamique de la relation à autrui qui conduit à sa refonte radicale, c'est-à-dire la profondeur de leur entente de la continuité affective/ émotionnelle du rapport indivis moi/autrui fondée sur un commun processus d'altérisation, qu'on le nomme couplage (Varela) ou croissance générative (Henry), et jusque la passivité radicale commune comme lieu de la relation, qu'il s'agisse d'une "épreuve de soi" ou d'une "dérive naturelle". ${ }^{4}$

1 M. Henry, "Pour une phénoménologie de la communauté ", dans Phénoménologie matérielle, Paris, PUF, "Epiméthée ", 1990, p. 197.

2 M. Henry, "Eux en moi", art. cit., p. 200.

3 bid., p. 205.

4 A propos de cette affinité remarquable entre les pensées de M. Henry et de F. J. Varela, N. Depraz a commencé à interroger leur "relation" lors d'une conférence donnée dans le cadre d'une séance 


\section{La phénoménologie «appliquée» de la vie : de la souffrance au soin}

Notre propos n'est pas ici, cependant, d'épuiser toutes les facettes de la rencontre "à venir" de ces deux penseurs magistraux du vivant (ce que j'ai commencé de faire ailleurs), mais de déployer plus largement l'intérêt et l'importance de la phénoménologie de la vie pour les approches en secondes personnes. À cet égard, la conception varelienne du couplage du vivant avec son environnement vient revisiter la relation henryenne à l'extériorité, au même moment où la conception henryenne de l'auto-affection, réciproquement, réinterroge la dynamique de l'auto-poièse du vivant. En effet, ce qui ressort moins dans la conception du vivant chez Varela, c'est le rôle des émotions, de l'affect et, plus largement, du souffrir comme pâtir, même si l'on a noté que la passivité comme dérive naturelle est loin d'être absente de son entente du vivant, et même s'il s'interrogeait également sur l'importance de l'affect. ${ }^{1}$

Pourtant, il n'en reste pas moins que toute la phénoménologie de la vie de M. Henry est bien plus crucialement orientée par l'épreuve du pâtir et du jouir à titre de nouds expérientiels complémentaires de la dynamique autoaffective du vivant. ${ }^{2}$ C'est ce qui, au-delà de la continuité ontologique structurelle de l'entente du vivant, laquelle permet à bon droit d'interrompre un instant la discontinuité méthodologique et épistémologique entre le régime empirique de la biologie et le régime transcendantal de la phénoménologie, fait entrer l'interrogation henryenne en résonance remarquable avec

(20 octobre 2009) du Séminaire "The Bodily Self and Its World" animé par D. Legrand au Centre de Recherche en Epistémologie Appliquée (CREA), intitulée "Auto-affection et auto-poièse. La non-rencontre de Michel Henry et de Francisco Varela". Il est à noter que Henry et Varela se sont côtoyés en 1997, mais jamais vraiment rencontrés; une autre occasion de rencontre "ratée" aurait correspondu au cycle de Conférences données en 2001 à Porto, où Henry a prononcé le texte aujourd'hui publié sous le titre: "Eux en moi : une phénoménologie", et où Varela figurait parmi les noms des personnes invitées mais, malheureusement, n'a pu être présent (communication personnelle de F. Martins), sans doute eu égard à son état de santé. Notons que N. Depraz compte prolonger et finaliser ce premier travail dans sa contribution au Colloque International Michel Henry: La vie et les vivants, qui se tiendra les 15 à 17 décembre prochains à l'Université catholique de Louvain.

1 F. Varela et N. Depraz, "At the source of time : valence and the constitutional dynamics of affect " dans S. Gallagher \& S. Watson (eds.), Ipseity and Alterity: Interdisciplinary Approaches to Intersubjectivity, Rouen, Presses Universitaires de Rouen, 2004; N. Depraz, "The Rainbow of emotions. At the crossroads between phenomenology and neurobiology", dans Continental Philosophy Research, 2008, 41, p. 237-259.

2 A cet égard, une autre résonance s'impose avec la pensée de M. Henry, dans le cadre de son dialogue manqué avec les sciences du vivant: le travail du neurobiologiste contemporain A. Damasio, à travers ses ouvrages référentiels, L'erreur de Descartes, Le sentiment même de soi et, très récemment, Spinoza avait raison. C'est l'objet d'une recherche en cours, qui s'inscrit pour commencer dans le cadre d'un Séminaire à l'Université de Rouen durant le Semestre 1, sous titre: "La Phénoménologie de la vie de M. Henry à la lumière des sciences cognitives: F. J. Varela et A. Damasion. 
un autre pan des sciences du vivant moins génériquement centré sur le vivant que tourné spécifiquement sur l'humain: nous voulons parler de la médecine et, plus particulièrement encore, de la psychiatrie, cette médecine remarquable de l'âme!

On l'a mentionné, Michel Henry est entré en dialogue à plusieurs reprises avec des médecins (souvent psychiatres), à Paris comme à Porto, durant les quelques dernières années de sa vie notamment. Après avoir consacré un long travail à la "généalogie de la psychanalyse", qui l'a conduit à un diagnostic critique vis-à-vis de la psychanalyse, il entre cette fois en sympathie avec l'approche psychiatrique des médecins qui, peut-on penser, résonne plus justement avec son intuition de l'épreuve radicale de soi et son sens aiguisé du souffrir originaire. Dans "Souffrance et vie", exemplairement, le phénoménologue, qui jamais ne renonce à ses postulats ontologiques ni ne se montre complaisant à l'égard des dangers voire des erreurs de la pratique scientifique souvent "réductionniste" (explicative), produit une phénoménologie de la souffrance qui résonne immédiatement avec des situations singulières empiriques que connaissent et traitent les praticiens hospitaliers. Au gré de son écriture dans ce texte, on voit apparaittre, après la reprise des thèses ontologiques bien connues (où s'opposent le monde et la vie, l'objectivité et l'invisibilités(I), une description saisissante du vécu de la souffrance (II). On peut reconstituer à mesure que l'analyse se déploie un certain nombre de traits caractéristiques de ce vécu : 1) le souffrir est global, la douleur liée à une "partie du corps objectif", même si, on l'a noté, les mots ne doivent pas être fils conducteurs figés, puisque le "douloureux comme tel" peut être global et la souffrance portée sur un point précis du corps ${ }^{4}$; 2) le souffrir adhère à lui-même, et se caractérise par l'absence absolue d'écart à soi ; 3) le souffrir est le terme générique de l'éprouver soi-même, qui répond à une pluralité de tonalités affectives diverses voire opposées (angoisses, plaisirs, désirs, bonheurs, tristesses, malaises, satisfactions, joies); 4) l'événement (accident, échec professionnel, maladie, deuil) n'est que l'occasion de l'expression du souffrir chez un être dont la constitution transcendantale inclut dès l'abord l'auto-affection ${ }^{5}$; 5) il y a une contemporanéité primitive du jouir et du souffrir, qui définissent à elles deux l'éprouver, et donnent lieu aux multiples tonalités négatives et positives de notre existence; 6) l'éprouver répond à la dynamique d'un passage incessant des tonalités les unes dans les autres, lesquelles ne sont ni isolables, ni bloquées en ellesmêmes. ${ }^{6}$ C'est ce vécu émotionnel du passage, de la transformation, qui

3 M. Henry, "Souffrance et vie", art. cit., p. 143-145.

4 Ibid., p. 145.

5 Ibid., p. 146-150.

6 Ibid., p. 151-152. 
conduit M. Henry, dans le troisième et dernier temps de cette conférence, ${ }^{1}$ à s'interroger sur ce moment-limite de bascule où le bonheur, fragile, se mue en malheur, où le souffrir se fait désespoir, puis dépression, se centrant ainsi sur des états émotionnels pathologiques dont l'émotion du désespoir forme le vecteur (dépression, décrite nommément, suicide, non-nommé explicitement, mais décrit à travers les expressions: "se défaire de soi", "vouloir échapper à sa souffrance", "se séparer de soi", "se détruire soi-même").

On pourrait s'en tenir là, et considérer cette description comme un prolongement naturel, "appliqué" à des vécus pathologiques de type psychiatrique, de la thèse henryenne de l'auto-affection comme souffrir originaire, ce qui permet, ce qui certes n'est pas rien, de montrer que la phénoménologie de la vie recèle une certaine fécondité pour la compréhension de l'approche en deuxième personne qui caractérise la psychiatrie, dès lors que celle-ci, comme c'est le cas dans sa version existentielle ou phénoménologique, s'intéresse au patient comme à un sujet qui souffre. Il y a là une continuité évidente avec la tradition de la Daseinsanalyse (Binswanger dans Mélancolie et Manie, de façon inaugurale, ou bien, plus récemment, Blankenburg, qui thématise avec la "perte de l'évidence naturelle" un vécu où le sujet s'échappe de lui-même et entre dans un état de dissociation).

Mais ce serait manquer radicalement l'originalité et l'inventivité de la parole henryenne. En effet, si l'auteur parle à un endroit de "thérapie ", ce n'est aucunement pour la présenter comme un effet nécessaire de la cause qu'est la maladie, ou encore comme un retour positif à la normalité, dans le cadre d'une volonté de guérir et de sortir d'un état pathologique jugé négatif, comme le comprenait encore la psychiatrie existentielle en opposant pathologie et normalité et en cherchant à guérir les patients de leur mal.

Que nous propose alors Michel Henry qui ne soit pas une guérison au sens d'une thérapeutique "ancienne manière"? De nombreuses formules dans cet article nous fournissent une partie de la réponse : (...) le vouloir se défaire de soi propre au désespoir se heurte en lui à la cohérence interne de son être, à l'essence infrangible de la vie. (...) au comble de sa souffrance, le désespoir se trouve renvoyé au souffrir primitif en lequel toute vie vient en soi, celle-ci est encore en lui, plus que jamais. (...) cette puissance est celle de la vie, à travers son souffrir, c'est l'irruption du vivre en lui-même qui s'accomplit. (...) en présence des maladies les plus graves qui affectent non pas seulement les corps mais la vie même, une thérapie est toujours possible, celle qui, d'une façon ou d'une autre, d'instinct ou délibérément, cherchera appui sur le mouvement inlassable en lequel la vie ne cesse de se

1 Ibid., p. 152-157.

2 Ibid., p. 155. 
donner à soi en se donnant à nous-mêmes. " ${ }^{1} \mathrm{Il}$ y a dans le souffrir la vitalité la plus aiguë depuis laquelle, comme un levier, la vie réinvestit le sujet de l'intérieur de lui-même jusqu'à le faire basculer de la tendance mortifère la plus affirmée au désir de s'en sortir le plus définitif. Au fond, ce que nous dit M. Henry, c'est que la souffrance contient en elle-même sa propre thérapie, dès que la première est prise au sérieux et regardée en face, estimée à sa juste mesure, bref, validée pour ce qu'elle est par le médecin, et non pas banalisée, passée à la trappe pour ménager le patient ou bien se voiler la face en refusant de voir la maladie en face. Dès lors, il ne s'agit pas tant de guérir au sens de sortir de la maladie que de se tenir en elle jusqu'à l'affronter de l'intérieur d'elle-même. C'est ce que fait le psychiatre urgentiste qui ne cherche pas à sortir le patient de la crise, mais, en le confrontant à celle-ci (passage à l'acte, délire), l'intensifie au contraire pour en révéler les mécanismes au patient et lui faire prendre conscience de la réalité profonde de sa situation. ${ }^{2}$

Ainsi, le soin est à puiser dans la souffrance elle-même, non en dehors d'elle, comme s'il fallait se débarrasser du mal pour pouvoir guérir et mieux vivre. C'est le constat extrêmement lucide de Raphaël Gély dans son article "Souffrance et attention sociale à la vie: Eléments pour une phénoménologie radicale du soin". Il y a une adhésion au souffrir qui conduit à subir la souffrance, c'est-à-dire à se résigner et à se perdre soi-même; il y a une adhésion au souffrir qui est consentement et acte, à savoir réaction génératrice de l'organisme vivant qui fait ainsi droit à la vie en lui. Cette adhésion plus profonde à la vie est le plan primitif de communication archaïque des ressentis entre le soignant et le soigné, d'où émerge la "partageabilité" de l'épreuve de la vie. ${ }^{3}$ Ainsi, une forme d'attention ouverte (vigilance), à savoir vivante à l'autre, distincte de l'attention focalisée, cognitive, à visée étroite (concentration) est ce qui préside à la possibilité de prendre soin d'autrui, non pas en sécurisant ce dernier, à savoir en le ménageant de façon mortifère, mais en vitalisant la relation. Or, on ne peut être vivifiant pour l'autre qu'en prenant également soin de soi, c'est-à-dire en ouvrant en soi-même l'espace d'une rencontre qui me décentre irréductiblement de moi-même. En effet, dans la vigilance, il y a la précarité d'une relation à autrui où le risque est support d'ouverture, et qui contraste avec la sécurité mortelle de la possession. Il y a là un antidote de taille à la perte de soi en jeu dans la dépression suicidaire, qui n'est pas l'opposé de la maladie, mais entre en

\footnotetext{
1 Ibid., p. 154-155.

2 Cf. à ce propos, F. Mauriac et N. Depraz, "Phénoménologie de la relation", art. cit., qui distingue la psychiatrie classique qui cherche à guérir par une thérapie et le travail relationnel de crise qui s'attache à intensifier celle-ci pour ouvrir le patient à la conscience la plus aiguë de ce qu'il vit, et lui donner des repères à même de l'aider à prendre appui sur son souffrir primitif comme un levier.

3 R. Gély, "Souffrance et attention sociale à la vie: Eléments pour une phénoménologie radicale du soin", dans Bulletin d'analyse phénoménologique, Volume 5, 2009, p. 14.
} 
continuité vertigineuse avec elle jusqu'à la retourner contre elle-même. Les quatre composantes principales de l'antidote attentionnel, le relationnel vs. l'individuel, l'ouverture vs. la clôture, la précarité vs. la possession, le risque vs. la sécurité définissent la cartographie opératoire d'une thérapeutique vécue, celle-là même que M. Henry appelle de son côté de ses vœux, en allant puiser dans le souffrir originaire de la dépression le levier même de la puissance vivifiante du sujet. Lantidote attentionnel est un tel levier. À cet égard, il faut pousser la provocation jusqu'à mettre au premier plan le soin de soi comme axe de cet antidote attentionnel: prendre soin de soi pour prendre soin d'autrui, c'est aller à l'encontre de deux modèles dominants, l'un, très connu, l'égocentrisme, l'autre, moins connu, l'altruisme. Comment cela? Le premier est bien connu: il prend ses marques dans toute une série de gestes philosophiques: le souci de soi (Heidegger, Foucault); l'ego comme centre de l'agir (éthiques de la décision); l'individu comme lieu exclusif de la connaissance (Kant); l'introspection ou l'auto-observation de ses pensées en psychologie; l'amour-propre, crucial dans les morales classiques. Laltruisme, son opposé apparent, est en fait un poison qui se fait passer pour un antidote; il se déploie à travers des mécanismes en réalité hautement toxiques, "pervers" pourrait-on dire, mais démasquables à partir des formules paradoxales suivantes: quand être généreux revient à se débarrasser d'autrui, quand être respectueux revient à ne pas s'impliquer, quand être poli revient à abandonner autrui, quand ménager l'autre revient à lui être indifférent voire à le mépriser. Ainsi, le souci, à la différence du soin, s'avère être un dénominateur commun de l'égocentrisme et de l'altruisme: être soucieux, avoir des soucis, c'est ne penser qu'à soi, et cette disposition entre en continuité étonnante (sous l'opposition apparente!) avec le fait de se faire du souci pour autrui, lequel revient à penser à autrui depuis soi, à savoir, à enfermer l'autre en soi.

Ainsi, le soin de soi est un véritable antidote au souci, et peut se décliner depuis des postures historiquement et culturellement hétérogènes, mais indicatrices d'une même authenticité: chez les Classiques, l'estime de soi est le levier de l'estime d'autrui (Montaigne: s'honorer soi-même; Rousseau: l'amour de soi); chez les Contemporains, prendre soin de soi, c'est se prendre soi-même comme un autre ${ }^{1}$; dans les traditions spirituelles, le soin indivis de soi-autrui se dit: "be gentle with yourself" (Ch. Trungpa), "l'ouverture du coeur" (Philocalie), "le centre ouvert en soi" ( ${ }^{\mathrm{me}}$ Guyon). Mais nous ne sommes pas au bout de nos peines, car le souci se fait parfois passer à notre insu pour du soin, et risque de nous induire en erreur. Ainsi de l'éthique du "care", dont les piliers tellement "éthiques", la place centrale de la relation entre l'agent et le patient, la responsabilité envers

1 P. Ricœur, Soi-même comme un autre, Paris, Editions du Seuil, 1990. 
l'autre vulnérable et dépendant, la sensibilité entendue comme sentiment et attention à l'autre, un ancrage dans le particulier, les situations concrètes de la vie plutôt que dans la généralité, ne peuvent que nous séduire, jusqu’à cette composante "féminine", qui s'oppose à une conception fondée sur l'autonomie, la justice, la norme ou encore l'universalité. ${ }^{1}$ Soit. Mais n'y a-t-il pas là une "sollicitude" un peu "naïve" qui nous fait retomber à notre insu dans l'altruisme insidieusement solidaire de l'égocentrisme le plus tenace? À cet égard, l'éthique de P. Ricour propose la distinction entre une "sollicitude naïve" (care) et "une sollicitude critique": le soi est ainsi fait qu'il peut (il a le droit) dans certains cas (de) faillir dans l'exercice de la sollicitude. Il y a alors nécessité du recours à la norme morale "caractérisée à la fois par la prétention à l'universalité et par un effet de contrainte ". ${ }^{2}$ En effet, quand la norme morale se trouve confrontée aux situations concrètes de la vie, des conflits moraux ne peuvent manquer de survenir. Dès lors, il y a une solution: une "sollicitude critique" qui "consiste à inventer les comportements justes appropriés à la singularité des cas "3. Conclusion: surtout ne pas "bien" prendre soin d'autrui, mais "mieux" prendre soin de lui, en s'éprouvant soimême (M. Henry) ou, variantes, en libérant autrui de moi-même (Sartre), en traitant autrui comme moi-même (Ch. Yannaras) - comme un invité de chaque soir -, c'est-à-dire, en se traitant comme un autre (Ch. Trungpa) - en découvrant l'ouverture qui est en soi-même. ${ }^{4}$

\section{La mise à l'épreuve de la vie auto-affectée dans un travail relationnel d'urgence psychiatrique}

Dans le temps précédent, nous avons montré comment la phénoménologie de la vie pouvait être "appliquée", dans le meilleur sens du terme, à des cas limites de risque suicidaire, et était en mesure d'apporter une lumière sur des situations concrètes extrêmes en instillant un mode thérapeutique de relation juste à ces situations "pathologiques". Nous avons vu également, de façon corollaire, combien l'éthique du soin qui accompagne une telle thérapeutique vécue, suppose une résonance avec le vécu interne du médecin, c'est-à-dire un soin de soi qui fasse levier et antidote aux apories croisées de l'égocentrisme individualiste et de l'altruisme bien pensant.

1 P. Paperman et S. Laugier (dir.), Le souci des autres. Ethique et politique du care, Paris, EHESS, 2005.

2 P. Ricour, op. cit., p. 200.

3 Ibid., p. 312.

4 Pour plus de précisions sur ce point, voir N. Depraz, "Promouvoir la vie, quels enjeux éthiques?", Conférence donnée à l'occasion de la cinquième Journée des pratiques soignantes, Centre Hospitalier Martin Charcot, 78, 18 mars 2010. Disponible en ligne en format Powerpoint. 
Nous voudrions à présent, pour accomplir ce mouvement de renouvellement de l'analyse philosophique au contact des pratiques empiriques, préciser la méthodologie qui nous paraît la plus idoine dans un tel contexte. En effet, jusque-là, nous avions parié sur l'auto-validité de l'analyse henryenne, sur son autonomie philosophique propre, depuis elle-même, à fournir des clés pour appréhender des situations singulières et les éclairer d'un nouveau jour, voire lui conférer une intelligibilité inouie. Nous nous sommes situés, en ce sens, dans le cadre d'une logique d'application dans le meilleur sens du terme, où prévaut le mode asymétrique de la relation du philosophique éclairant unilatéralement l'empirique. Ceci correspond à la posture-standard du philosophe permettant au scientifique de comprendre le sens de ce qu'il fait. Est-ce aussi simple que cela?

Nous cherchons dans ce dernier temps à faire émerger un mouvement inverse, en réalité déjà présent dès le début de cette enquête, et que l'on peut formuler ainsi: la pratique empirique donne des clés au philosophe, permet aux concepts de recevoir une intelligibilité concrète qui leur fait défaut en tant que tels. Ainsi, c'est notre intuition initiale, exprimée à l'orée de ce propos à partir de la situation concrète de ce jeune homme de vingtdeux ans qui souhaite mourir et à qui le médecin psychiatre répond en osant lui dire qu'il a le droit de mourir plutôt que de le ménager, de le protéger de son désir suicidaire en cherchant à le rassurer et à le réconforter (ce qui n'aurait fait qu'accentuer, par opposition, son souhait de mourir), qui nous a permis de donner sens à l'affirmation henryenne de la puissance en dernière instance vitalisante du souffrir originaire, lequel trouve au fond de lui-même - de sa dépression la plus profonde - le ressort interne de sa vitalisation ultime. En elle-même, l'analyse de M. Henry reste générale, et sa relation à des expériences singulières toujours illustratives d'une vérité générique, jamais motrices d'une analyse: les exemples sont fugaces, les allusions expérientielles non détaillées. Tandis que le philosophe ne prend appui que de façon lacunaire sur des situations singulières en première personne, l'exemple que nous allons présenter pour conclure, et que nous avons d'ailleurs présenté dès le départ pour avérer la circularité de sa relation avec l'analyse conceptuelle, a un caractère heuristique et renouvelant. Ainsi, notre hypothèse est que la pratique parle d'elle-même et contribue à renouveler l'analyse philosophique, ce qui, pour autant, n'inféode pas unilatéralement cette dernière à la première: il y a bel et bien co-générativité des deux plans expérientiel (empirique) et catégoriel (transcendantal).

Nous allons, à partir de la description détaillée de l'échange qui naît au moment même de la crise dans laquelle se trouve ce jeune homme éprouvé par un délire suicidaire, faire émerger le mécanisme de vitalisation de la relation qui s'enclenche à même ce délire dramatique et donne naissance 
comme en osmose au geste thérapeutique. Un jeune homme de vingt-deux ans, marié, un enfant, éducateur spécialisé en formation. Son cri unique, tout au long de l'entretien: "Laissez-moi mourir, je veux mourir!" La réponse du thérapeute: "Je pourrais lui dire: "Monsieur, vous avez la liberté de vous suicider, vous êtes libre de vous suicider" ". La liberté telle que je l'exprime est "contrainte", car, dans ma position, un patient en menace suicidaire peut s'y sentir abandonné; en effet, ma position de professionnel, paradoxalement, laisse seul le patient: celui-ci se sentira quitté et, ainsi, se quittera effectivement lui-même. Alors, je lui dis: "Vous avez le droit de vous suicider. " La différence peut sembler ténue, une nuance qui, cependant, fait passer de la mort à la vie. En disant cela, je lui fais vivre sa liberté de choisir de mourir, ce qui lui fait éprouver un espace de liberté qu'il n'est plus seul à s'octroyer. Il peut alors se sentir respecté; je lui donne ainsi la possibilité de dialoguer sans avoir besoin de se faire violence pour trouver sa place ultime. Etant donné que son problème est bien là: vouloir se suicider, c'est déjà choisir la solution extrême pour avoir le dernier mot.

En effet, avoir le dernier mot par la mort, c'est la quête ultime de quelqu'un de non respecté. C'est ici que l'on peut saisir la profondeur du sens du propos de R. Gély: la naturalisation du désir de vie enchaîne; il y a de la gravité à sortir de cette naturalisation de la vie en redonnant la liberté au sujet avec son "pathique" ultime: le droit de choisir de mourir. Ce qui est déterminant, au-delà du fait d'avoir raison (en mode raisonnable) sur la nécessité de rester en vie, c'est de créer une relation avec ce patient suicidaire. En posant la relation avant l'évidence de rester vivant, on a une chance de lui permettre de s'autoriser à lui-même de maintenir le lien avec la vie.

Ainsi, la réponse non henryenne au patient serait du type: "Mais non, c'est bien de vivre, il faut vous ressaisir!", tandis que la réponse henryenne se formulerait ainsi: "Je pense que vous avez raison de vouloir mourir dans votre situation dramatique, vous avez le droit ", ce qui met l'accent sur sa responsabilité indirecte, à savoir enclenche un processus interne spontané de responsabilisation, tandis que: "Vous êtes libre de mourir" est une réponse abandonnante qui ne génère pas la vie et ne permet pas le ressaisissement interne du patient.

\section{Conclusion}

La mise en résonance de la pensée de Michel Henry avec des paroles thérapeutiques concrètes en situation de crise est-elle pertinente? La parole du phénoménologue de la vie, qu'on a montré si consonante avec le travail 
relationnel empirique des praticiens urgentistes, est-elle en elle-même intelligible si l'on n'a pas soi-même traversé une épreuve de cette teneur et adopté a minima les outils pratiques relationnels inhérents à une épreuve? Comment saisir, à cet égard, la subtilité de son insistance sur une thérapie vécue, qui entre en phase remarquable avec l'urgence d'une non-thérapie dans le travail de crise, au sens d'un souci de ne pas guérir, c'est-àdire recouvrir et masquer le mal par le bien, pour surtout faire émerger l'intensité du problème et le manifester en toute lumière aux yeux et à la conscience du patient, lequel seul saura le travailler de l'intérieur de l'intensité de sa crise suicidaire?

"Voulant se défaire de soi, le désespéré veut mourir mais ce vouloir est contradictoire et ne parvient pas à ses fins. Vouloir mourir sans pourtant mourir, mourir sa mort de telle façon que c'est là plutôt "vivre sa mort" (...) Plus grande est la détresse en laquelle, désespérant de soi et voulant se défaire de soi, le moi mesure son impuissance à se détruire lui-même, plus violente et plus forte aussi l'épreuve qu'il fait de la puissance invincible qui le jette en lui-même en dépit de son vouloir, indépendamment de son pouvoir. Mais cette puissance est celle de la vie, à travers son souffrir c'est l'irruption du vivre en lui-même qui s'accomplit "'. C'est M. Henry qui parle. Notre méthode, certes, est différente de celle du phénoménologue de la vie, car nous partons de la productivité créatrice de l'empirique, de sa nouveauté motrice et heuristique, laquelle est assurément en résonance avec les intuitions philosophiques de M. Henry, mais les renouvellent tout aussi bien, à savoir les déplacent et les reformulent autrement. On voit dans cette configuration empirique émerger de nouvelles catégories, éthico-politiques, dont celle du droit de mourir opposé à la liberté de mourir. Nous avons avancé une mise en relation entre l'ontologie de la vie de M. Henry et l'éthique de la sollicitude critique de P. Ricœur. Ce lien demanderait à être davantage exploré, en prolongeant peut-être la médiation pratico-pratique de cette situation de crise, mais ce serait la matière d'une autre contribution.

Natalie Depraz et Fréderic Mauriac

1 M. Henry, "Souffrance et vie ", art. cit., p. 154-155. 